\title{
COMMUTATORS, GENERALIZED EIGENFUNCTION EXPANSIONS AND SINGULAR INTEGRAL OPERATORS $\left({ }^{1,2}\right)$
}

This paper is dedicated to the memory of John Williams Calkin.

BY

JOEL DAVID PINCUS

\section{INTRODUCTION}

Suppose that $U$ and $V$ are bounded symmetric operators on a Hilbert space $H$, and suppose that $U V-V U=(i / \pi) P$, where $P$ is a projection onto a one-dimensional subspace of $H$. Assume, further, that the smallest closed invariant subspace of $U$ containing the range of $P$ is $H$ itself. Under these conditions it is known through the work of Xa-Dao-Xeng [13] and C. R. Putnam [14] that both $U$ and $V$ have absolutely continuous spectral resolutions. We are able to achieve a much more detailed analysis of the situation, and we will show how it is possible to explicitly construct a complete diagonalization of $V$ from that of $U$, i.e. we find all the invariant subspaces of $V$.

The result is equivalent to the construction of the generalized eigenfunction expansion associated with the (symmetrizable) singular integral operator

$$
\operatorname{Lx}(\lambda)=A(\lambda) x(\lambda)+\frac{1}{\pi i} \mathrm{P} \int_{a}^{b} \frac{k(\mu)}{\mu-\lambda} x(\mu) d \mu
$$

having bounded measurable real coefficients $A(\lambda)$ and $k(\lambda)$. Accordingly, the main point of the present work is to extend the author's previous results giving the explicit diagonalization of singular integral operators of the above and related forms when the coefficients $A(\lambda)$ and $k(\lambda)$ are differentiable $[11]\left({ }^{3}\right)$.

In order to achieve this end, we shall describe a method, which seems to be new, for constructing the absolutely continuous part of self-adjoint operators and their associated Direct-Integral-Diagonalizing-Hilbert spaces in terms of an object which we will call a splitting form.

1. The case of simple multiplicity. Let $\mathscr{H}$ be a Hilbert space and let the self-adjoint operator $L$ defined on $\mathscr{H}$ have simple multiplicity. By definition,

Received by the editors November 16, 1964 and, in revised form, January 12, 1965.

(1) This paper is a revised version of On the construction of generalized eigenfunction expansions, Brookhaven National Laboratory Rep. No. 7963 (January, 1964).

(2) Work performed under the auspices of the U.S. Atomic Energy Commission.

(3) This reference will henceforth be denoted by I. 
there is a vector $k \in \mathscr{H}$ such that vectors of the form $f(L) k$, where $f(v)$ is a Borel function defined over the spectrum of $L$, are dense in $\mathscr{H}$.

Let $E_{\lambda}$ denote the spectral resolution of $L$, then

THEOREM 1. If $L$ is absolutely continuous, i.e. if the set function $\mu(\Delta)=\left\|E_{\Delta} k\right\|^{2}$ is absolutely continuous with respect to Lebesgue measure, then the operator $\mathscr{S}$ on $\mathscr{H}$ defined on those elements of $\mathscr{H}$ that can be written as finite linear combinations $q=\sum a_{i}\left(L-\omega_{i}\right)^{-1} k$ by

$$
\mathscr{S}_{q}=\frac{1}{2 \pi i} \frac{1}{\beta_{k}(\xi)} \lim _{n \downarrow 0}\left(q,\left[(L-\xi+i \eta)^{-1}-(L-\xi-i \eta)^{-1}\right] k\right),
$$

where

$$
\beta_{k}(\xi)=\frac{d\left\|E_{\xi} k\right\|^{2}}{d \xi}
$$

and through closure everywhere else, gives a spectral representation for $L$. That is, if $g=f(L) k$, then $\mathscr{S} g=f$ so that

$$
f(L) k=\int_{\sigma(L)} \mathscr{S} f(L) k(v) d E_{v} k .
$$

The proof is a simple consequence of the Hilbert resolvent identity. We first establish

LemMa 1.

$$
\mathscr{S}(L-\omega)^{-1} k=(M-\omega)^{-1} \mathscr{S} k,
$$

where $\omega \notin \sigma(L)$, and $M$ denotes the operator of multiplication by the spectral variable of $L$.

Proof. Let us note that

$$
(L-\xi \pm i \eta)^{-1}(L-\omega)^{-1}=\frac{\xi \pm i \eta-\omega}{(\omega-\xi)^{2}+\eta^{2}}\left[(L-\xi \pm i \eta)^{-1}-(L-\omega)^{-1}\right]
$$

so that

$$
\begin{aligned}
\mathscr{S}(L-\omega)^{-1} k(\xi)= & \lim _{\eta \downarrow 0} \frac{\xi-\omega}{(\omega-\xi)^{2}+\eta^{2}} \mathscr{S} k(\xi) \\
& +\frac{1}{2 \pi} \frac{1}{\beta_{k}(\xi)} \lim _{\eta \downarrow 0} \frac{\eta}{(\omega-\xi)^{2}+\eta^{2}}\left[\left((L-\xi+i \eta)^{-1} k, k\right)\right. \\
& +\frac{1}{\pi \beta_{k}(\xi)} \lim _{\eta \downarrow 0} \frac{\eta}{(\omega-\xi)^{2}+\eta^{2}}\left((L-\omega)^{-1} k, k\right) .
\end{aligned}
$$

Since $\omega \notin \sigma(L)$, the limit of the last term is zero, and only the second term remains. 
However,

$$
\begin{aligned}
& \left((L-\xi+i \eta)^{-1} k, k\right)+\left((L-\xi-i \eta)^{-1} k, k\right) \\
& \quad=\int_{\sigma}\left(\frac{1}{v-\xi+i \eta}+\frac{1}{v-\xi-i \eta}\right) \beta_{k}(v) d v
\end{aligned}
$$

and since $\int_{\sigma} \beta_{k}(v) d v=\|k\|^{2}$, a theorem of A. Plessner [5] shows that this expression has a finite limit almost everywhere, and hence the lemma is established.

It follows from the lemma and the easily established fact that $\mathscr{S} k(\xi)=1$, that $\mathscr{S}(L-\omega)^{-1} k(\xi)=1 /(\xi-\omega)$. The spectral theorem then shows us that $\mathscr{S}$, considered as a transformation from $\mathscr{H}$ to $L_{2}(d \mu)$ preserves the norm of any finite sum of the form: $\sum a_{i}\left(L-\omega_{i}\right)^{-1} k$. Accordingly, $\mathscr{S}$ can be uniquely extended to an isometric transformation defined on all of $\mathscr{H}$, which we again call $\mathscr{S}$.

To conclude the proof of the theorem we must establish that $\mathscr{S} f(L) k(\xi)=f(\xi)$ for arbitrary $f(\cdot) \in L_{2}(d \mu)$.

Since the continuous functions are dense in $L_{2}(d \mu)$, this result will be established if we can show that it is possible to choose a sequence $\left\{\omega_{i}\right\}$ of complex numbers for which finite linear combinations of the functions $\left\{1 /\left(\xi-\omega_{i}\right)\right\}$ are dense in the continuous functions with respect to the $L_{2}(d \mu)$ norm. For this purpose we recall that S. N. Bernštein [6] has shown that any continuous function can be uniformly approximated by such sums (plus a constant) over the whole real axis. Thus, if $g(\xi)$ is a continuous function, then $\left(\xi-\omega_{1}\right) g(\xi)$ is also continuous; and we can choose constants $\left\{B_{k}\right\}$ so that

$$
\max _{-\infty<\xi<\infty}\left|\left(\xi-\omega_{1}\right) g(\xi)-B_{1}-\sum_{k=1}^{N} \frac{B_{k}}{\xi-\omega_{k}}\right|<\varepsilon
$$

for some $N_{1}$ and thus there are constants $\left\{C_{k}\right\}$ such that

$$
\max _{-\infty<\xi<\infty}\left|g(\xi)-\sum_{k=1}^{N} \frac{C_{k}}{\xi-\omega_{k}}\right|-\frac{\varepsilon}{\left|\xi-\omega_{1}\right|} .
$$

Hence,

$$
\begin{aligned}
\int_{-\infty}^{\infty}\left|g(\xi)-\sum_{k=1}^{N} \frac{C_{k}}{\xi-\omega_{k}}\right|^{2} d \mu(\xi) & <\varepsilon^{2} \int_{-\infty}^{\infty} \frac{d \mu(\xi)}{\left|\xi-\omega_{1}\right|^{2}} \\
& =\varepsilon^{2}\left\|\left(L-\omega_{1}\right)^{-1} k\right\|^{2} \text {. q.e.d. }
\end{aligned}
$$

THEOREM 2. Consider the manifold $M$ in $L^{2}(d \mu)$ of finite linear combinations $\sum a_{i} /\left(\xi-\omega_{1}\right)$. Define $\mathscr{T}$ for $g \in M$ by

$$
\mathscr{T} g(\lambda)=-\frac{1}{2 \pi i} \lim _{\eta \downarrow 0} \int_{\sigma(L)} \frac{\overline{H(\xi+i \eta, \lambda)}-\overline{H(\xi-i \eta, \lambda)}}{\beta_{k}(\xi)} g(\xi) d \mu(\xi),
$$

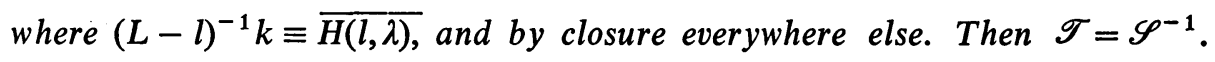


Proof. It suffices to show that $\mathscr{T}(\xi-\omega)^{-1}=H(\omega, \lambda)$. But, since $\sigma(L)$ is closed and the resolvent of $L$ is continuous on the complement of $\sigma(L)$, we can write

$$
\mathscr{T}\left(\frac{1}{\xi-\omega}\right)=\frac{1}{2 \pi i} \lim _{\eta \downarrow 0} \int_{-\infty}^{\infty} \frac{d \xi}{\xi-\omega} \int_{\sigma(L)}\left[\frac{1}{v-\xi-i \eta}-\frac{1}{v-\xi+i \eta}\right] d E_{v} K .
$$

If we make use now of the Lebesgue dominated convergence theorem for vector valued measures [2, p. 328], by first writing the $d \xi$ integral as the limit of integrals from $-N$ to $N$ and then writing each of these integrals as a Riemann sum, and finally exchanging the two limits with the $d E_{v} k$ integration, we will have

$$
\mathscr{T}\left(\frac{1}{\xi-\omega}\right)=\frac{1}{2 \pi i} \lim _{\eta \downarrow 0} \int_{\sigma(L)} d E_{v} k \int_{-\infty}^{\infty} \frac{d \xi}{\xi-\omega}\left[\frac{1}{v-\xi-i \eta}-\frac{1}{v-\xi+i \eta}\right] .
$$

A standard estimate shows $[7$, p. 445$]$ that we can invoke the scalar dominated convergence theorem and the Plemelj-Privalov [5] theorem to get

$$
\begin{aligned}
\mathscr{T}\left(\frac{1}{\xi-\omega}\right) & =\int_{\sigma(L)} d E_{v} k \frac{1}{2 \pi i} \lim _{\eta \downarrow 0} \int_{-\infty}^{\infty} \frac{d \xi}{\xi-\omega}\left[\frac{1}{v-\xi-i \eta}-\frac{1}{v-\xi+i \eta}\right] \\
& =\int_{\sigma(L)} \frac{d E_{v} k}{v-\omega}=H(\omega, \cdot) . \quad \text { q.e.d. }
\end{aligned}
$$

REMARK. The above theorem tells us that the transformation inverse to $\mathscr{S}_{k}$ is given by the complex conjugated kernel (as expected). It is, however, possible to to express $\mathscr{S}_{k}$ and $\mathscr{S}_{k}^{-1}$ in another and far more useful way; namely,

$$
\mathscr{S}_{k} f(\xi)=-\frac{1}{2 \pi i} \lim _{\eta \downarrow 0} \int \frac{F_{k}(\xi, \lambda+i \eta)-F_{k}(\xi, \lambda-i \eta)}{\beta_{k}(\xi)} f(\lambda) d \lambda
$$

where, by definition,

$$
F_{k}(\xi, z) \equiv \beta_{k}(\xi) \mathscr{S}_{k}\left(\frac{1}{\lambda-z}\right)(\xi)
$$

This is immediate $\left({ }^{4}\right)$ since

$$
\begin{aligned}
\frac{1}{2 \pi i} & \lim _{\eta \downarrow 0} \int \mathscr{S}_{k}\left(\frac{1}{s-\lambda+i \eta}-\frac{1}{s-\lambda-i \eta}\right) f(\lambda) d \lambda \\
& =\frac{1}{2 \pi i} \mathscr{S}_{k}\left(\lim _{\eta \downarrow 0} \int\left(\frac{1}{s-\lambda+i \eta}-\frac{1}{s-\lambda-i \eta}\right)\right) f(\lambda) d \lambda \\
& =\mathscr{S}_{k} f(\xi) .
\end{aligned}
$$

This formula expresses the equality

$$
F_{k}(\xi, \lambda+i 0)-F_{k}(\xi, \lambda-i 0)=H_{k}(\xi+i 0, \lambda)-H_{k}(\xi-i 0, \lambda) .
$$

(4) This was shown in a rather complicated way in I. 
2. Higher multiplicity. If $L$ is not simple, we can decompose $\mathscr{H}$ into pairwise orthogonal invariant subspaces $\mathscr{H}_{\boldsymbol{k}_{i}}$, determined by a choice of vectors $\left\{k_{i}\right\}$, on each of which $L$ is simple. Note that it is an easy consequence of the Hilbert resolvent identity that $\mathscr{S}_{j}\left(\mathscr{H} \ominus \mathscr{H}_{j}\right)=0$ when $\mathscr{S}_{j}$ is extended to all $\mathscr{H}$.

Definition. We define the bilinear splitting form associated with a self-adjoint operator $L$ by

$$
P_{\xi}[f, g]=\frac{1}{2 \pi i} \lim _{\eta \downarrow 0}\left(f,\left[(L-\xi-i \eta)^{-1}-(L-\xi+i \eta)^{-1}\right] g\right) .
$$

THEOREM 3.

$$
P_{\xi}[f, g]=\sum_{j=1}^{m(\xi)}\left(\mathscr{S}_{j} f\right)(\xi) \overline{\left(\mathscr{S}_{j} g\right)(\xi)} \beta_{j}(\xi)=\frac{\partial}{\partial \xi}\left(E_{\xi} f, g\right),
$$

where $m(\xi)$ denotes the spectral multiplicity of $\xi \in \sigma(L)$, and $P_{\xi}[f, g]$ vanishes when $f$ or $g$ belongs to the orthogonal complement of the domain of the absolutely continuous part of $L$.

To prove this theorem we choose the sequence $\left\{k_{i}\right\}$ to give a minimal HellingerHahn decomposition of $\mathscr{H}=\Sigma \oplus \mathscr{H}_{\boldsymbol{k}_{i}}$. Then we use the Plemelj formula and the fact that the projections $P_{i}$ onto $\mathscr{H}_{k_{i}}$ sum to the identity, commute with $L$, and are orthogonal.

Specialization to the case that $\mathscr{H}=L_{2}(R)$.

Definition. Let us define the splitting kernel of $L$ to be

$$
P_{\xi}(x, y) \equiv P_{\xi}\left[\frac{1}{\lambda-x}, \frac{1}{\lambda-y}\right]=\frac{\partial}{\partial \xi}\left(E_{\xi} \frac{1}{\lambda-x}, \frac{1}{\lambda-y}\right)
$$

when $x$ and $y$ are not real. Then, if we let

$$
\mathscr{S}_{j}\left(\frac{1}{\lambda-x}\right)=\frac{F_{j}(\xi, x)}{\beta_{j}(\xi)},
$$

we will have, by Theorem 3 :

$$
P_{\xi}(x, y)=\sum_{j=1}^{m(\xi)} \frac{F_{j}(\xi, x) \overline{F(\xi, y)}}{\beta_{j}(\xi)} .
$$

LEMMA. $\quad F_{j}(\xi, x)$ was really defined as a one-parameter family of vectors $L_{2}\left(d \mu_{k}\right)$. We assert that it is possible to construct a function of $\xi, F_{j}(\xi, x)$ with the property that $F_{j}^{\prime}(\xi, x)=F_{j}(\cdot, x)$ when considered as a vector in $L_{2}\left(d \mu_{k}\right)$, while for almost all fixed $\xi, F_{j}^{\prime}(\xi, x)$ is analytic in $x$ for $\operatorname{Im} x \neq 0$.

Proof. Gelfand and Kostjutschenko [12] have shown that if $L$ is a self-adjoint operator in $L^{2}\left(R^{N}\right)$, then the functional $\partial E_{\xi} k / \partial \mu_{k}(\xi)$ exists for almost all $\xi$ as a continuous linear functional on a space $\Phi$, formed from the infinitely differentiable 
square integrable functions, $\phi$ on $E^{n}$ under the norm $\|\phi\|_{1}=\max (|D \phi| M(\lambda))$, where $\left(\left|\lambda_{1} \cdots \lambda_{n}\right|\right)^{1 / 2} / M(\lambda)$ is square integrable, while $D=\partial^{n} / \partial \lambda_{1} \cdots \partial \lambda_{n}$.

It suffices to take $M(\lambda)=\prod_{i=1}^{n}\left(1+\left|\lambda_{i}\right|\right)^{3 / 2+\varepsilon}$ with $\varepsilon>0$ when $\mathscr{H}=L_{2}\left(R^{n}\right)$. Then we will have $\left\|\prod\left(\lambda_{i}-z_{i}\right)^{-1}\right\|_{\Phi_{1}}<\infty$. But the definition of $F_{k}(\xi, z)$ given above and the Plessner $L^{1}$ version of the Plemelj-Primalov formulae show that fo fixed $x$

$$
F_{k}(\xi, x)=\frac{1}{\beta_{k}(\xi)} \frac{\partial}{\partial \xi}\left(E_{\xi} k, \prod_{1}^{n}\left(\frac{i}{2 \pi}\right)^{n-1} \frac{1}{\lambda_{i}-x_{i}}\right)
$$

almost everywhere in $\xi$. Thus, our assertion follows if we take

$$
F_{k}^{\prime}(\xi, x)=\frac{1}{\beta_{k}(\xi)}\left(\frac{\partial}{\partial \xi} E_{\xi} k, \prod_{1}^{n}\left(\frac{i}{2 \pi}\right)^{n-1} \frac{1}{\lambda_{i}-x_{i}}\right),
$$

i. e. if we apply the continuous linear functional $\partial / \partial \xi E_{\xi} k$ to the indicated element in its domain of continuity. Since

$$
\begin{gathered}
\left\|\frac{\partial}{\partial z_{j}} \prod_{i=1}^{n} \frac{1}{\lambda_{t}-x_{i}}\right\|_{\Phi_{1}}<\infty, \\
\frac{\partial}{\partial z_{j}} F_{k}^{\prime}(\xi, x)=\frac{1}{\beta_{k}(\xi)} \frac{\partial}{\partial \xi}\left(E_{\xi} k\left(\frac{i}{2 \pi}\right)^{n-1} \frac{\partial}{\partial z_{j}} \prod_{i=1}^{n} \frac{1}{\lambda_{i}-x_{i}}\right),
\end{gathered}
$$

a.a. $\xi$ and the assertion is proved.

We henceforth drop the prime on $F_{k}^{\prime}(\xi, z)$.

Definition. We define the $j$ th Determining Function of $L$, by

$$
E_{j}(l, z)-1=\frac{1}{2 \pi i}\left((L-l)^{-1} k_{j^{\prime}}, \frac{1}{\lambda-\bar{z}}\right),
$$

where $l \notin \sigma(L)$ and $\operatorname{Im} z \neq 0$.

The Plemelj-formula then gives us:

$$
F(\xi, z)=E(\xi+i 0, z)-E(\xi-i 0, z) \quad \text { a.a. } \xi \in \sigma(L) \forall z
$$

with $\operatorname{Im} z \neq 0$.

Now we turn to

3. The direct integral representation space $\mathscr{H}^{*}$. The aim of the present section is to construct a new direct integral space $\mathscr{H}^{*}$, isometric to $\mathscr{H}$, which diagonalizes $L$. ( $\mathscr{H}^{*}$ will be a space of analytic functions.)

By working in this space it is rather simple to recover the essentials of the Hellinger-Hahn [10] multiplicity theory, and in particular to give an apparently new characterization of the spectral multiplicity function [10], $m(\xi)$, of $L$.

Finally, we will show how to construct an ordered set of generalized eigenfunctions $\left\{\omega_{j}^{\prime}(\xi, \lambda)\right\}$ with the property that $\omega_{j}^{\prime}(\xi, \lambda) \equiv 0$ for $j>m(\xi)$. 
We now work with a decomposition sequence $\left\{k_{i}\right\}$ which is not necessarily minimal.

Let $\left\{\phi_{n}\right\}$ be a complete orthonormal set in $\mathscr{H}$, and let $\mu_{n}(\xi)=\left\|E_{\xi} \phi_{n}\right\|^{2}$. Let $a_{1}, a_{2}, \cdots$ be a sequence of positive numbers with $\sum a_{n}<\infty$. Then $\sigma(\xi)=\sum a_{n} \mu_{n}(\xi)$ is equivalent to Lebesgue measure since $\sigma>\mu_{n}, \forall n$ [10] and each set of Lebesgue measure zero has $\mu_{n}$ measure zero. $\sigma(\xi)$ has the useful property that $\int_{-\infty}^{\infty} d \sigma(\xi)<\infty$.

The function $P_{\xi}(x, y)$ is obviously a positive matrix function and may be regarded as the reproducing kernel [9] of an associated Hilbert space $\mathscr{H}_{\xi}$ generated from the finite linear combinations of the form

$$
g(\xi, x)=\sum_{k=1} \alpha_{k} P_{\xi}\left(x, y_{k}\right)
$$

with the inner product

$$
\left(\left(g, g^{\prime}\right)\right)=\sum_{k=1}^{m} \alpha_{i} \bar{\alpha}_{k}^{\prime} P_{\xi}\left(y_{k}, y_{i}\right)
$$

The Hilbert space $\mathscr{H}_{\xi}$ obtained by taking the closure in the norm topology derived from this scalar product is characterized by the property that $h(y)=\left(\left(h(x), P_{\xi}(x, y)\right)\right)$ for all $h(y) \in \mathscr{H}_{\xi}$.

This reproducing property of the kernel $P_{\xi}(x, y)$ in turn enables us to conclude:

LeMma (a). The nonidentically zero $F_{j}(\xi, z) /\left(\beta_{j}(\xi)\right)^{1 / 2} \equiv G_{j}(\xi, z)$ form a complete orthonormal set in $\mathscr{H}_{\xi}$.

Proof.

$$
\begin{aligned}
G_{k}(\xi, y) & =\left(\left(G_{k}(\xi, x), \underset{j}{\sum_{j}} G_{j}(\xi, x) \overline{\left.\left.G_{j}(\xi, y)\right)\right)}\right.\right. \\
& =\sum_{j} G_{j}(\xi, y)\left(\left(G_{k}(\xi, y), G_{j}(\xi, x)\right)\right)
\end{aligned}
$$

and

$$
\left(\left(G_{k}, G_{l}\right)\right)=\sum_{j}\left(\left(G_{j}, G_{l}\right)\right)\left(\left(G_{k}, G_{j}\right)\right)
$$

so that

$$
\left\|G_{k}\right\|_{\mathscr{H}_{\xi}}^{2}=\sum_{j}\left|\left(\left(G_{j}, G_{k}\right)\right)\right|^{2} \text {. }
$$

Thus $\left(\left(G_{j}, G_{k}\right)\right)=0$ if $j \neq k$, while the first relation of the proof implies that $\left\|G_{k}(\xi)\right\|^{2}=1$ when $G_{k}(\xi)$ is not a null-vector. q.e.d.

We will see below that the dimension, $m(\xi)$, of $\mathscr{H}_{\xi}$ is the spectral multiplicity function of $L$, i.e., the function entering the definition of the von Neumann direct integral space associated with $L[10]$. 
We form a new Hilbert space $\mathscr{H}^{*}$. The elements of $\mathscr{H}^{*}$ are (the equivalence classes of) those functions $g(\xi, z)$ that can be written $g(\xi, z)=\sum \alpha_{j}(\xi) G_{j}(\xi, z)$, where each $\alpha_{j}(\xi)$ is measurable with $\int \sum\left|\alpha_{j}(\xi)\right|^{2} d \sigma(\xi)<\infty$, and the scalar product in $\mathscr{H}^{*}$ is given by

$$
\left(g, g^{\prime}\right)=\int \sum \alpha_{j}(\xi) \overline{a_{j}^{\prime}(\xi)} d \sigma(\xi)
$$

THEOREM 4. $\mathscr{H}^{*}$ is the direct integral $[10]$ of the $\mathscr{H}_{\xi}$ with respect to $\sigma$, i.e. $\mathscr{H}^{*}=\int \oplus \mathscr{H}_{\xi} d \sigma(\xi)$.

Proof. For fixed $\xi$ we write $g(\xi, z)=g_{z}(\xi)$ when $g(\xi, z) \in \mathscr{H}^{*}$, and we set $g(\xi, z)=\int g_{z}(\xi)(d \sigma(\xi))^{1 / 2}$. We call such functions summable, and ask: are there any other functions satisfying the two requirements:

(1) For every $\sigma$-summable $g(\xi),((f(\xi), g(\xi))$ is $\sigma$-measurable.

(2) If $\|f(\xi)\|_{\mathscr{H}_{\xi}}^{2}$ is $\sigma$-measurable, then $\int\|f(\xi)\|_{\mathscr{H}_{\xi}}^{2} d \sigma(\xi)<\infty$ ?

Since the two requirements (1) and (2) characterize the summable functions, a negative answer to the question will establish the theorem.

Suppose that $f(\xi) \in \mathscr{H}_{\xi}$ and satisfies (1) and (2).

Now, since $f(\xi) \in \mathscr{H}_{\xi} ; f(\xi)=\sum f_{j}(\xi) G_{j}(\xi, z)$ where $\Sigma\left|f_{j}(\xi)\right|^{2}<\infty$, and if $g(\xi) \in \mathscr{H}_{\xi}$ we have

$$
((f(\xi), g(\xi)))=\sum f_{j}(\xi) \overline{g_{j}(\xi)} .
$$

However, since this must hold for all $g(\xi, z)$ of our form, including that $g(\xi, z)$ with

$$
g_{j}(\xi)= \begin{cases}1 & j=k, \\ 0 & j \neq k,\end{cases}
$$

we can conclude that $f_{j}(\xi)$ is measurable. But $\int\|f(\xi)\|_{\mathscr{H}_{\xi}}^{2} d \sigma(\xi)<\infty$ if and only if $\int \Sigma\left|f_{n}(\xi)\right|^{2} d \sigma(\xi)<\infty$. Thus we have chosen a definition of summability which implies that $\mathscr{H}^{*}=\int \oplus \mathscr{H}_{\xi} d \sigma(\xi)$.

THEOREM 5. $\mathscr{H}^{*}$ is isometrically isomorphic with $\mathscr{H}$ and furnishes a spectral representation for $L$.

Proof. Let $f \in \mathscr{H}$, and define $\hat{f}(\xi, z) \leftrightarrow f$ by

$$
\hat{f}(\xi, z)=\sum_{j}\left(\beta_{j}(\xi) \frac{d \xi}{d \sigma}\right)^{1 / 2} \mathscr{S}_{j} f(\xi) G_{j}(\xi, z) .
$$

We see that

$$
\|\hat{f}(\xi, z)\|_{\mathscr{H} *}^{2}=\int \Sigma\left|\mathscr{S}_{j} f(\xi)\right|^{2} d \mu_{j}=\left\|f_{\mathrm{d}}\right\|_{\mathscr{H}}^{2},
$$

while, on th other hand, if $\hat{g}(\xi, z)=\Sigma_{j} g_{j}(\xi) G_{j}(\xi, z) \in_{-}^{*} \mathscr{H}^{*}$, we have 


$$
\begin{aligned}
\int\left|g_{j}(\xi)\right|^{2} d \sigma & =\int\left|g_{j}(\xi)\left(\frac{d \sigma}{d \xi}\right)^{1 / 2}\right|^{2} d \xi \\
& =\int\left|g_{j}(\xi)\left(\frac{1}{\beta_{j}(\xi)} \frac{d \sigma}{d \xi}\right)^{1 / 2}\right|^{2} d \mu_{j}(\xi)<\infty, \quad j=1,2, \cdots,
\end{aligned}
$$

and thus can form

$$
\mathscr{S}_{j}^{-1}\left(g_{j}(\xi)\left(\frac{1}{\beta_{j}(\xi)} \frac{d \sigma}{d \xi}\right)^{1 / 2}\right)=f_{j} \in \mathscr{H}_{j}
$$

and $f=\sum f_{i}$.

Since $\mathscr{H}^{*}$ is the direct integral of the $\mathscr{H}_{\xi}$, it is possible to choose $\left({ }^{5}\right)$ a family of functions $\Phi_{1}(\xi, z), \Phi_{2}(\xi, z), \cdots$ in $\mathscr{H}^{*}$ with the property that they form a complete orthonormal set in $\mathscr{H}_{\xi}$.

It is then easily seen (by the preceding remark) that the distributions

$$
\omega_{r}^{\prime}(\xi, \lambda)=\frac{1}{2 \pi i}\left(\Phi_{r}(\xi, \lambda+i 0)-\Phi_{r}(\xi, \lambda-i 0)\right)
$$

form an ordered set of generalized eigenfunctions.

In this way we can recast the multiplicity theory of Hellinger-Hahn in terms of eigenfunction expansions.

4. Commutators and singular integrals. Let $U$ and $V$ be bounded symmetric operators on a Hilbert space $\mathscr{H}$, and suppose that $U V-V U=(i / \pi) P$, where $P$ is a projection onto a one-dimensional subspace of $\mathscr{H}$. Assume, further, that the smallest closed invariant subspace of $U$ containing $R(P)$, the range of $P$, is $\mathscr{H}$ itself.

Let $E_{\lambda}$ denote the spectral resolution of $U$, and take $e \in R(P)$ so that $P x=e(x, e)$. Set $\sigma(\lambda)=\left(\hat{E}_{\lambda} e, e\right), \lambda \in[a, b] \equiv \sigma(U)$. It is known that $\sigma(\lambda)$ is absolutely continuous [13], thus if $W$ denotes the diagonalizing transformation of $U$ (constructed, for example, by the method embodied in equation (1)) we will have $W e=1 \in L_{2}(d \sigma)$. Now Xa-Dao-Xeng [13] has shown that

$$
L f \equiv \frac{1}{i} W V W^{-1} f(\lambda)=A(\lambda) X(\lambda)+\frac{1}{\pi i} \mathrm{P} \int_{a}^{b} \frac{k(\mu) f(\mu)}{\mu-\lambda} d \mu,
$$

where $k(\mu)=\sigma^{\prime}(\mu)$ is essentially bounded, and $A(\lambda)$ is a real bounded measurable function obtained by setting

$$
q(\lambda)=W V W^{-1} 1 \in L_{2}(d \sigma) \text { and } A(\lambda)=\operatorname{Re}[q(\lambda)]
$$

(5) A proof of this assertion is given in Theorem 1 of [10], the method is to choose a complete orthonormal set it $\mathscr{H}^{*}$ and then to orthogonalize its projections in each $\mathscr{H}_{\xi}$ by Gram-Schmidt orthogonalization. 
Now we return to the construction of the invariant subspaces of $V$.

5. Singular integral operators with bounded measurable coefficients. In this consider

$$
L x(\lambda)=A(\lambda) x(\lambda)+\frac{1}{\pi i} \mathrm{P} \int_{a}^{b} \frac{k(\mu) x(\mu)}{\mu-\lambda} x(\mu) d \mu .
$$

$L$ is a self-adjoint operator in $L_{2}(d \sigma)$ and a symmetrizable operator in $L_{2}(a, b)$; note that a very slight modification of the results to be obtained will suffice for the reduction of

$$
L_{1}=A(\lambda) x(\lambda)+\frac{1}{\pi i} \mathbf{P} \int_{a}^{b} \frac{k_{1}(\lambda) k_{2}(\mu)}{\mu-\lambda} x(\mu) d \mu,
$$

where $A(\lambda), k_{1}(\lambda), k_{2}(\lambda)$ are bounded real and measurable, and for the reduction of

$$
L_{2}=A(\lambda) x(\lambda)+\frac{1}{\pi i} \mathbf{P} \int_{a}^{b} \frac{\phi(\lambda) \overline{\phi(\mu)}}{\mu-\lambda} x(\mu) d \mu
$$

where $\phi(\lambda)$ is bounded and measurable on $[a, b]$.

We may assume that $k(\lambda) \neq 0$ a.e. on $[a, b]$.

The operator $L_{1}$ defined above, considered as an operator on $L_{2}(R)$, is not self-adjoint. Hence, the theory developed in $\S \S 2$ and 3 can not be applied directly.

However, $L_{1}$ is symmetrizable [15]. If we set

$$
L_{s} x(\lambda)=A(\lambda) x(\lambda)+\frac{1}{\pi i} \mathbf{P} \int_{a}^{b} \frac{\left(k_{1}(\lambda) k_{2}(\mu) k_{1}(\mu) k_{2}(\lambda)\right)^{1 / 2}}{\mu-\lambda} x(\mu) d \mu
$$

then

$$
L_{s} y(\lambda)=\left(\frac{k_{2}(\lambda)}{k_{1}(\lambda)}\right)^{1 / 2} L_{1}\left(\frac{k_{1}(\cdot)}{k_{2}(\cdot)}\right)^{1 / 2} y(\cdot)
$$

with a corresponding simple relation between the unitary transformation $U$ diagonalizing $L_{s}$ and the nonunitary transformation $W$ diagonalizing $L_{1}$; namely

$$
W=U\left(\frac{k_{2}(\cdot)}{k_{1}(\cdot)}\right)^{1 / 2}
$$

The form of the diagonalizing transformation for such nonsymmetric singular integral operators (with smooth coefficients) was already given in I, and we can easily see from the preceding sections in what sense symmetrizable operators on $L_{2}(R)$ in general possess generalized eigenfunction expansions.

We now turn to the task of analyzing the operator $L$, above. This operator is self-adjoint in $L_{2}(d \sigma)$, and we may apply the theory of $\S \S 1-3$ except that we replace all scalar products in $L_{2}(R)$, by scalar products in $L_{2}(d \sigma)$.

Assume, for the moment that the coefficients $A(\lambda)$ and $k(\lambda)$ are smooth and satisfy the hypothesis of I. Then we will have the 
LEMMA.

where

$$
\begin{aligned}
E(l, z)-1 & \equiv \frac{1}{2 \pi i}\left((L-l)^{-1}[2 k(\lambda)], \frac{1}{\lambda-z}\right) L_{2}(d \sigma) \\
& =\exp \left(\frac{1}{2 \pi i} \int_{a}^{b} \log \frac{A(\mu)-l-k(\mu)}{A(\mu)-l+k(\mu)} \frac{d \mu}{\mu-z}\right)-1 \\
& =\exp \left(\frac{1}{2 \pi i} \int_{a}^{b} \int_{\sigma} g(v, \mu) \frac{d \mu}{\mu-z} \frac{d v}{v-l}\right)-1 \\
& \equiv \hat{E}(l, z)-1
\end{aligned}
$$

$$
g(v, \mu)=\frac{1}{\pi} \arg \frac{A(\mu)-v-i 0-k(\mu)}{A(\mu)-v-i 0+k(\mu)} .
$$

Proof. By the representation theorem on p. 118 of I and a result on line 16 of p. 112 of I we see that (using the notation of that reference)

$$
\mathscr{S}_{0}(2 k(\lambda))=B(\xi), \quad \mathscr{S}_{0}\left(\frac{k(\lambda)}{\lambda-z}\right)=\frac{\overline{F(\xi, \bar{z})}}{B(\xi) / 2^{1 / 2}}
$$

where $B(\xi)>0$ a.a. $\xi \in \sigma(L)$. Thus, using the fact that $\mathscr{S}$ furnishes a spectral representation of $L$ on the smallest invariant subspace of $H$ containing $k(\lambda)$, we have

$$
\frac{1}{2 \pi i}\left(2 k(\lambda),(L-l)^{-1} \frac{k(\lambda)}{\lambda-\bar{z}}\right)_{L_{2}(k)}=\frac{1}{2 \pi i} \int_{\sigma(L)} \frac{F(\xi, z)}{\xi-l} d \xi,
$$

where $F(\xi, z)=\hat{E}(\xi+i 0, z)-\hat{E}(\xi-i 0, z)$.

Thus, the lemma is proved for smooth coefficients. In fact, however, the lemma is true if the coefficients are only bounded and measurable, as will become clear from the approximation argument carried out below.

Let us choose $A^{(n)}(\lambda)$ and $k^{(n)}(\lambda) \in C^{1}(a, b)$, satisfying the hypothesis of the representation theorem of $I$ and such that

$$
\lim _{n \rightarrow \infty} \int_{a}^{b}\left|A^{(n)}(\lambda)-A(\lambda)\right|^{2} d \lambda+\int_{a}^{b}\left|k^{(n)}(\lambda)-k(\lambda)\right|^{2} d \lambda=0 .
$$

Thus, the operators

$$
L^{(n)} x(\lambda)=A^{(n)}(\lambda) x(\lambda)+\frac{1}{\pi i} \mathrm{P} \int_{a}^{b} \frac{k^{(n)}(\mu)}{\mu-\lambda} x(\mu) d \mu
$$

fulfill the requirements of previous work, and determine a sequence of splitting kernels $P^{(n)}(x, y)$.

We will show that the limit operator $L$ has an absolutely continuous spectrum, and the above sequence of splitting kernels not only converges-but converges to the splitting kernel of the limit operator.

The results of the previous section show that this result corresponds to the complete diagonalization of $L$. 
THEOREM 6.

$$
\lim _{n \rightarrow \infty} P_{\xi}^{(n)}(x, y)=P_{\xi}(x, y), \quad \text { a.a. } \xi \in \sigma(L), \operatorname{Im} x, \operatorname{Im} y \neq 0 .
$$

The proof rests upon certain identities which we exhibit in the form of a series of lemmas.

LEMMA.

where

$$
P_{\xi}^{(n)}(x, y)=\sum_{j=1}^{m(\xi)} \frac{F_{j}^{(n)}(\xi, x) \overline{F_{j}^{(n)}(\xi, y)}}{\beta_{j}(\xi)}
$$

$$
\begin{aligned}
& F_{j}^{(n)}(\xi, x)=E^{(n)}(\xi+i 0, x)-E^{(n)}(\xi-i 0, x), \\
& F_{j}^{(n)}(\xi, x)=\frac{F_{j}^{(n)}(\xi, x)}{x-\lambda_{j}^{(n)}(\xi)}, \quad 1<j \leqq m(\xi),
\end{aligned}
$$

and $\lambda_{j}^{(n)}(\xi)$ is the jth root of $F_{1}^{(n)}(\xi, x)$, while the $\beta_{j}^{(n)}(\xi)$ are certain positive quantities which will be given later.

Proof. This lemma is an immediate consequence of Theorem 3 of this paper and the representation theorem of $I$.

It is possible to derive another expression for the approximate splitting kernels, and we turn now to this task. We may set

$$
\log \prod \frac{T_{j}^{(n)}(\xi)-z}{S_{j}^{(n)}(\xi)-z}=\frac{1}{\pi} \int_{a}^{b} \arg \frac{A^{(n)}(\mu)-\xi-i 0-k^{(n)}(\mu)}{A^{(n)}(\mu)-\xi-i 0+k^{(n)}(\mu)} \frac{d \mu}{\mu-z}
$$

since the hypothesis of the representation theorem of I imply that there can be at most a finite number of intervals, $m(\xi)$, of $\mu,\left[T_{j}^{(n)}(\xi), S_{j}^{(n)}(\xi)\right]$, for each $\xi \in \sigma\left(L_{\xi}\right)$ for which the "index function"

$$
\frac{1}{\pi} \arg \frac{A^{(n)}(\mu)-\xi-i 0-k^{(n)}(\xi)}{A^{(n)}(\mu)-\xi-i 0+k^{(n)}(\xi)}=1
$$

Then, if we define $T^{(n)}(v, z)=\prod\left[T_{j}^{(n)}(v)-z\right]$ and $S^{(n)}(v, z)=\prod\left[S_{j}^{(n)}(v)-z\right]$, and set

we $\operatorname{get}\left({ }^{6}\right)$

$$
Q(\xi, x, y)=\frac{T(v, x) S(v, y)-T(v, y) S(v, x)}{[S(v, x)-T(v, x)][S(v, y)-T(v, y)]}
$$

$$
\begin{aligned}
\frac{1}{x-y} Q^{(n)}(\xi, x, y)= & \sum_{1}^{m(\xi)-1} \frac{\left(u_{j}^{(n)}(\xi)\right)^{1 / 2}}{y-\lambda_{j}^{(n)}(\xi)} \frac{\left(u_{j}^{(n)}(\xi)\right)^{1 / 2}}{x-\lambda_{j}^{(n)}(\xi)} \\
& +\frac{1}{\sum_{1}^{m(\xi)-1}\left[T_{j}^{(n)}(\xi)-S_{j}^{(n)}(\xi)\right]}
\end{aligned}
$$

(6) These notations were developed in I. 
where the simplicity of the poles $\lambda_{j}(\xi)$ follows from: $S_{1}^{(n)}(\xi) \leqq T_{1}^{(n)}(\xi) \leqq S_{2}^{(n)}(\xi)$ $\leqq \cdots S_{P}^{(n)}(\xi) \leqq T_{P}^{(n)}(\xi)$, as does the positivity of the residues $\mu_{j}(\xi)$.

Now, using the representation theorem of I again, we see that the quantities $\beta_{j}^{(n)}(\xi)$ of the preceding lemma are given by

$$
\begin{aligned}
& \beta_{1}^{(n)}(\xi)=\frac{1}{2\left[\sum_{1}^{m(\xi)-1}\left[T_{j}^{(n)}(\xi)-S_{j}^{(n)}(\xi)\right]\right]}, \\
& \beta_{q}^{(n)}(\xi)=\frac{\mu_{q}^{(n)}(\xi)}{2}, \quad 1<j \leqq m(\xi) .
\end{aligned}
$$

LEMMA.

$$
P_{\xi}^{(n)}(x, \bar{y})=E^{(n)}(\xi+i 0, x) \overline{E^{(n)}(\xi-i 0, y)}-E^{(n)}(\xi-i 0, x) \overline{E^{(n)}(\xi+i 0, y) .}
$$

The proof follows from the identity $\left({ }^{7}\right)$

$$
\begin{aligned}
Q^{(n)}(\xi, x, \bar{y}) F^{(n)}(\xi, x) \overline{F^{(n)}(\xi, y)}= & E^{(n)}(\xi+i 0, x) \overline{E^{(n)}(\xi-i 0, y)} \\
& -E^{(n)}(\xi-i 0, x) \overline{E^{(n)}(\xi+i 0, y)}
\end{aligned}
$$

which, in turn, comes from the representation

$$
E^{(n)}(l, z)=\exp \left(\frac{1}{2 \pi i} \int_{\sigma} \log \frac{T^{(n)}(v, z)}{S^{(n)}(v, z)} \frac{d v}{v-l}\right)
$$

which implies that $T^{(n)}(v, z) E^{(n)}(v+i 0, z)-S^{(n)}(v, z) E^{(n)}(v-i 0, z)=0$.

Now, by this lemma we have

$$
\begin{aligned}
\lim _{n \rightarrow \infty} P_{\xi}^{(n)}(x, y) \\
\quad=\lim \left[! E^{(n)}(\xi+i 0, x) \overline{E^{(n)}(\xi-i 0, y)}-E^{(n)}(\xi-i 0, x) \overline{E^{(n)}(\xi+i 0, y)}\right] .
\end{aligned}
$$

Thus, the heart of the theorem is found in the

LemMa.

\section{Proof.}

$$
\begin{array}{r}
\lim _{\eta \downarrow 0} \lim _{n \rightarrow \infty} E^{(n)}(\xi \pm i \eta, z)=\lim _{n \rightarrow \infty} \lim _{\eta \downarrow 0} E^{(n)}(\xi \pm i \eta, z) \\
\text { for } \xi \in R, \text { and } \operatorname{Im} z \neq 0 .
\end{array}
$$

$$
\begin{aligned}
\log E^{(n)}(l, z) & =\frac{1}{2 \pi i} \int_{\sigma\left(L^{n}\right)} \log \frac{T^{(n)}(v, z)}{S^{(n)}(v, z)} \frac{d v}{v-l} \\
& =\frac{1}{2 \pi^{2} i} \int_{-\infty}^{\infty} \int_{a}^{b} \arg \left(\frac{A^{(n)}(\mu)-v-i 0-k^{(n)}(\mu)}{A^{(n)}(\mu)-v-i 0+k^{(n)}(\mu)}\right) \frac{d \mu}{\mu-z} \frac{d v}{v-l} .
\end{aligned}
$$

Let us take the $n$-limit first. Since the function

(7) Proved in I. 


$$
\arg \left(\frac{A^{(n)}(\mu)-v i 0-k^{(n)}(\mu)}{A^{(n)}(\mu)-v-i 0+k^{(n)}(\mu)}\right)
$$

is bounded by $\pi$ independently of $n$, we can use the Lebesgue dominated convergence theorem to pass the limit on $n$ underneath the double integral. Then, using the Plemelj formula, we can take the $\eta$-limit to get

$$
\begin{array}{r}
\frac{1}{\pi} \mathbf{P} \int_{-\infty}^{\infty} \int_{a}^{b} \arg \left(\frac{A(\mu)-v-i 0-k(\mu)}{A(\mu)-v-i 0+k(\mu)}\right) \frac{d \mu}{\mu-z} \frac{d v}{v-z} \\
\pm \frac{1}{\pi} \int_{a}^{b} \arg \left(\frac{A(\mu)-\xi-i 0-k(\mu)}{A(\mu)-\xi-i 0+k(\mu)}\right) \frac{d \mu}{\mu-z} .
\end{array}
$$

Let us now evaluate the result of interchanging the order of the limits. If we take the limit on $\eta$ first we set

$$
\begin{array}{r}
\frac{1}{\pi} \mathbf{P} \int_{-\infty}^{\infty} \int_{a}^{b} \arg \left(\frac{\left(A^{(n)}(\mu)-v-i 0-k^{(n)}(\mu)\right.}{A^{(n)}(\mu)-v-i 0+k_{(n)}(\mu)}\right) \frac{d \mu}{\mu-z} \frac{d v}{v-z} \\
\pm \frac{1}{\pi} \int_{a}^{b} \arg \left(\frac{A^{(n)}(\mu)-\xi-i 0-k^{(n)}(\mu)}{A^{(n)}(\mu)-\xi-i 0+k^{(n)}(\mu)}\right) \frac{d \mu}{\mu-z} .
\end{array}
$$

But the finite Hilbert transformation is a bounded operator in $L_{2}(a, b)$ [17]. Hence, we can take the limit in $n$ underneath the integrals to get the same result as before. q.e.d. Thus, we have shown that

$$
\begin{aligned}
\lim _{n \rightarrow \infty} & \lim _{\eta \downarrow 0} P_{\xi}^{(n)}(x, y) \\
& \left.=\lim _{\eta \downarrow 0} \lim _{n \rightarrow \infty}\left(\frac{1}{\lambda-x},\left(L^{(n)}-\xi-i n\right)^{-1}-L^{(n)}-\xi+i n\right)^{-1} \cdot \frac{1}{\lambda-y}\right)
\end{aligned}
$$

and the theorem will be proved if we can show that

LEMMA.

$$
\lim _{n \rightarrow \infty}\left(L_{s}^{(n)}-l\right)^{-1} \frac{1}{\lambda-y}=\left(L_{s}-l\right)^{-1} \frac{1}{\lambda-y}, \quad \operatorname{Im} l \neq 0 .
$$

Proof. The Carleman solution theory [16] for singular integral equations reduces the solution problem to solving a Hilbert barrier relation in the complex plane. It suffices to consider the unsymmetric operator $L$ now rather than $L_{s}$. By the standard solution theory we get, for $g(\lambda) \in L_{2}(a, b)$

$$
\begin{aligned}
\left(L^{(n)}-l\right)^{-1} g(\lambda) & =E^{(n)}(l, \lambda+i 0) R^{(n)}(l, \lambda+i 0)-E^{(n)}(l, \lambda-i 0) R^{(n)}(l, \lambda-i 0), \\
(L-l)^{-1} g(\lambda) & =E(l, \lambda+i 0) R(l, \lambda+i 0)-E(l, \lambda-i 0) R(l, \lambda-i 0), \operatorname{Im} l \neq 0,
\end{aligned}
$$

where

$$
R^{(n)}(l, \lambda)=\frac{1}{2 \pi i} \int_{a}^{b} \frac{k^{(n)}(\mu) g(\mu)}{\left[A^{(n)}(\mu)-l+k^{(n)}(\mu)\right]} \frac{1}{E^{(n)}(l, \mu+i 0)} \frac{d \mu}{\mu-z}
$$


and

$$
R(l, \lambda)=\frac{1}{2 \pi i} \int_{a}^{b} \frac{k(\mu) g(\mu)}{[A(\mu)-l+k(\mu)]} \quad \frac{1}{E(l, \mu+i 0)} \frac{d \mu}{\mu-z} .
$$

But the Lebesgue dominated convergence theorem allows us to assert that $\lim _{n \rightarrow \infty} R^{(n)}(l, \lambda)=R(l, \lambda)$, and hence the lemma, and with it the theorem, is proved. q.e.d.

With this construction of the splitting kernel of $L$ we have succeeded in completely diagonalizing the operator $V$ of the introduction.

We will, however, explicitly construct the generalized eigenfunctions of $V$ by computing the $F_{j}(\xi, z)$ which give rise to them. In doing this we call attention to the existence of a new phenomenon associated with the occurrence of infinite multiplicity that was excluded by the hypothesis of [1].

\section{The Generalized Eigenfunctions}

We turn now to a study of the important function

Since

$$
\Theta(v, z)=\exp \left\{\frac{1}{\pi} \int_{a}^{b} \arg \frac{A(\mu)-v-i 0-k(\mu)}{A(\mu)-v-i 0+k(\mu)} \frac{d \mu}{\mu-z}\right\}
$$

$$
\frac{1}{\pi} \arg \frac{A(\mu)-v-i 0-k(\mu)}{A(\mu)-v-i 0+k(\mu)}, \text { for fixed } v,
$$

is the characteristic function of a bounded measurable set, we may assert that

$$
\Theta(v, z)=1+\int_{a}^{b} \frac{d \mu_{v}(\lambda)}{\lambda-z} .
$$

Where $d \mu_{v}(\lambda)$ is a uniquely determined singular $\left({ }^{8}\right)$ measure (i.e. the sum of a purely discrete measure and a singularly continuous measure) for which

$$
\int_{a}^{b} d \mu_{v}(\lambda)=\frac{1}{\pi} \int_{a}^{b} \arg \frac{A(\mu)-v-i 0-k(\mu)}{A(\mu)-v-i 0+k(\mu)} d \mu .
$$

Now let us consider the poles of the related function

$$
L(v, z) \equiv \frac{1}{1-\Theta(v, z)},
$$

i.e. the zeros of

$$
\int_{a}^{b} \frac{d \mu_{v}(\lambda)}{\lambda-z}
$$

LEMMA. There is a nonnegative measure $d M_{v}(\mu)$ on the Borel subsets of the line and a number $A_{v} \geqq 0$ such that, for $\operatorname{Im} z>0$

(8) The fact that $d \mu_{v}(\lambda)$ is singular follows from a result on p. 386 of [19]. 
furthermore,

$$
\frac{d}{d z} L(v, z)=\int_{a}^{b} \frac{d M_{v}(\mu)}{(\mu-z)^{2}}+A_{v}
$$

$$
\int_{a}^{b} \frac{d M_{v}(\mu)}{1+\mu^{2}}<\infty
$$

\section{Proof.}

$$
\operatorname{Im} L(v, z)=\frac{\operatorname{Im} \Theta(v, z)}{|1-\Theta(v, z)|^{2}} .
$$

However, $\operatorname{Im} e^{\omega}=e^{\mathrm{Re} \omega} \sin \operatorname{Im} \omega$; hence, the $\operatorname{sign}$ of $\operatorname{Im} L(v, z)$ is determined by the sign of

$$
\sin \frac{y}{\pi} \int_{a}^{b} \arg \frac{A(\mu)-v-i 0-k(\mu)}{A(\mu)-v-i 0+k(\mu)} \frac{d \mu}{(\mu-x)^{2}+y^{2}}
$$

when $z=x+i y$. But it is well known that

$$
\frac{y}{\pi} \int_{a}^{b} \frac{\phi(\mu)}{(\mu-x)^{2}+y^{2}} d \mu \leqq \operatorname{ess} \sup |\phi(\mu)| ;
$$

hence, we are evaluating the sine of a positive quantity bounded by $\pi$, and we conclude that $\operatorname{Im} L(v, z)$ has positive imaginary part in the upper half plane.

Accordingly, we may invole the Poisson representation theorem to assert that there is a non negative measure $d M_{v}(\mu)$ on the Borel sets of the real line su ch that $\int_{-\infty}^{\infty} d M_{v}(\mu) /\left(1+\mu^{2}\right)<\infty$ and a constant $A_{v} \geqq 0$ such that for $y>0$

$$
\operatorname{Im} L(v, z)=\int_{-\infty}^{\infty} \frac{y d M_{v}(\mu)}{(\mu-x)^{2}+y^{2}}+A_{v} y .
$$

From this we may conclude, by differentiation with respect to $x$ and then with respect to $y$ together with the Cauchy-Riemann equations, that

$$
\frac{d}{d z} L(v, z)=\int_{-\infty}^{\infty} \frac{d M_{v}(\mu)}{(\mu-z)^{2}}+A_{v} . \quad \text { q.e.d. }
$$

As a simple consequence we have the

LEMMA. The poles of $L(v, z)$ are real and simple.

But the results of the preceding sections can be used to give a proof of

LEMMA.

$$
P_{\xi}(x, \bar{y})=\frac{F(\xi, x) \overline{F(\xi, \bar{y}})}{x-y}\left\{\frac{1}{1-\Theta(v, x)}-\frac{1}{1-\Theta(v, y)}\right\}
$$

Hence, we may write 


$$
\begin{array}{r}
P_{\xi}(x, \bar{y})=F(\xi, x) \overline{F(\xi, \bar{y})}\left\{\frac{1}{A(\xi)}+\sum_{j=1}^{m(\xi)-1} \frac{\mu_{j}(\xi)}{\left(x-\lambda_{j}(\xi)\right)\left(y-\lambda_{j}(\xi)\right)}\right. \\
\left.\quad+\int_{a}^{b} \frac{d M_{\xi}^{s}(\mu)}{(\mu-x)(\mu-y)}\right\},
\end{array}
$$

where $\lambda_{j}(\xi)$ denotes the $j$ th pole of $L(v, z)$. [The ordering is chosen according to the comment after Theorem 5.] $\mu_{j}(\xi)$ is the positive mass which the measure $d M_{\xi}(\mu)$ assigns to $\lambda_{j}(\xi)$, and $d M_{\xi}^{s}(\mu)$ is the continuous part of $d M_{\xi}(\mu)$.

The last contribution above occurs whenever the spectral point $\xi$ has infinite multiplicity. It occurs because an infinite number of poles $\lambda_{j}(\xi)$ occur in the finite interval $(a, b)$ and, hence, have a limit point which is an essential singularity of $L(v, z)$.

The generalized eigenfunctions of $L$ are therefore formed by the jump across the real axis of the functions

$$
\frac{F_{1}(\xi, z)}{\left(B_{1}(\xi)\right)^{1 / 2}}=\frac{F(\xi, z)}{(A(\xi))^{1 / 2}}, \frac{F_{j}(\xi, z)}{\left(B_{j}(\xi)\right)^{1 / 2}}=\frac{F(\xi, z)}{z-\lambda_{j}(\xi)}\left(\mu_{j}(\xi)\right)^{1 / 2}
$$

together with the jumps of $\left\{F(\xi, z) G_{\infty}^{(\alpha)}(\xi, z)\right\}$, when the spectrum has infinite multiplicity, where

$$
\left.Q_{\infty}(\xi, x, \bar{y}) \equiv \int_{a}^{b} \frac{d M_{\xi}^{s}(\mu)}{(\mu-x)(\mu-y)}=\sum_{\alpha} G_{\infty}^{(\alpha)}(\xi, x) \overline{G_{\infty}^{\alpha}(\xi, \bar{y}}\right)
$$

and the existence of such a bilinear decomposition follows from the results of $\S 3$ applied to the positive kernel $Q_{\infty}(\xi, x, y)$.

Of course, we also note that it is possible to gain a better insight into the structure of these generalized eigenfunctions by amalgamating both the sequences

$$
\left\{\frac{1}{z-\lambda_{j}(\xi)}\right\} \text { and }\left\{G_{\infty}^{(\alpha)}(\xi, z)\right\}
$$

according to the following obvious consequence of the Parseval equality:

LemMA. Let $\left\{P_{\xi}^{(k)}(\mu)\right\}$ be a complete orthonormal set in $L_{2}\left(d M_{\xi}(\mu)\right)$. Then

$$
\int \frac{d M_{\xi}(\mu)}{(x-\mu)(\bar{y}-\mu)}=\sum_{k} \int \frac{P_{\xi}^{(k)}(\mu)}{\mu-x} d M_{\xi}(\mu) \overline{\int \frac{P_{\xi}^{(k)}(\mu)}{\mu-y} d M_{\xi}(\mu) .}
$$

From this result, we immediately deduce that

LEMMA. The spectral multiplicity, $m(\xi)$, is equal to one plus the dimension of $L_{2}\left(d M_{\xi}(\mu)\right)$.

REMARK. The measure $d M_{\xi}(\mu)$ is purely singular. 
We write

$$
\begin{aligned}
\lim _{n \rightarrow \infty} \int \frac{d M_{\xi}^{(n)}(\mu)}{\mu-z}+1 & =\int \frac{d M_{\xi}(\mu)}{\mu-z}+1 \\
& =\lim _{n \rightarrow \infty} \exp \left\{\int \frac{f_{\xi}^{(n)}(x)}{x-z} d x\right\}=\exp \left\{\int \frac{f_{\xi}(x)}{x-z} d x\right\},
\end{aligned}
$$

where the measures $d M_{\xi}^{(n)}(\mu)$ are determined from the functions $A^{(n)}(\lambda)$ and $k^{(n)}(\lambda)$ used before, and, thus, the $f_{\xi}^{(n)}(x)$ are characteristic functions of a disjoint collection of intervals. The assertion now follows from the cited theorem of Aronszajn and Donoghue.

It may be appropriate to remark here that exactly the same techniques suffice to treat the operators $L_{1}, L_{2}$, and $L_{s}$. However, in order to treat $L_{2}$ it is convenient to take $P_{\xi}[\phi(\lambda) /(\lambda-x), \phi(\lambda) /(\lambda-y)]$ instead of $P_{\xi}[1 /(\lambda-x), 1 /(\lambda-y)]$ as the splitting kernel, $P_{\xi}(x, y)$.

With this choice, the second lemma of the present section remains valid, and a complete theory of the operator $L_{2}$ is obtained by step by step duplication of the procedure for the operator $L$.

REMARK. The last lemma immediately allows us to extend the index characterization of multiplicity given for the smooth operators in I (p. 108) as follows

For fixed $\xi$ consider the index function of $L$,

$$
g(\xi, \mu)=\frac{1}{\pi} \arg \frac{A(\mu)-\xi-i 0-k(\mu)}{A(\mu)-\xi-i 0+k(\mu)}
$$

or that of $L_{2}$,

$$
\frac{1}{\pi} \arg \frac{A(\mu)-\xi-i 0-|k(\mu)|^{2}}{A(\mu)-\xi-i 0+|k(\mu)|^{2}} .
$$

Consider the set of $\mu$ 's for which the index does not vanish. If that set is a union of $n_{\xi}$ disjoint intervals then the multiplicity is equal to $n_{\xi}$; otherwise, it is infinite. In summary, we have explicitly constructed a diagonal representation for the operators $L, L_{1}, L_{s}$, and $L_{2}$. That is, we have constructed the spectrum of these operators and direct integral Hilbert spaces $\mathscr{H}^{*}$ characterized in terms of explicitly computed multiplicity functions, $m(\xi)$, together with the spectral transformations (and their inverses) mapping $L_{2}(a, b)$ onto $\mathscr{H}^{*}$ in terms of a sequence of $m(\xi)$ integral operators whose kernels are generalized eigenfunctions of our operators.

The theory developed in $\S \S 1-3$ could be properly employed for our singular integral operators because we know by [14] that these operators have absolutely continuous spectra.

However, it is not necessary to rely on the cited work and, in fact, it is useful for many purposes to give the following criterion for absolute continuity of an operator. 
THEOREM 7. The self-adjoint operator $L$ has an absolutely continuous spectrum if and only if

$$
\int_{\sigma} P_{\xi}[f, f] d \xi=\|f\|^{2} \quad \text { for each } f \in \mathscr{H} .
$$

Proof. By Theorem 3, if $f$ belongs to the domain of the absolutely continuous part of $L$

$$
\int_{\sigma} P_{\xi}[f, f] d \xi=\int_{\sigma} \frac{\partial}{\partial \xi}\left(E_{\xi} f, f\right) d \xi=\|f\|^{2}
$$

To prove the converse implication, let us write $H=\Sigma \oplus \mathscr{H}_{\boldsymbol{k}_{\boldsymbol{i}}}$ where there is, by assumption, at least one $k_{j}$ for which the measure $\left\|E_{\Delta} k_{j}\right\|^{2}$ is not absolutely continuous. Then if we let $P_{i}$ denote the orthogonal projection of $H$ onto $\mathscr{H}_{i}$ while $\mathscr{S}_{i}$ denotes the diagonalizing transformation of $L$ on $\mathscr{H}_{i}$ whose existence is given by the spectral theorem for self-adjoint operators (and which is explicitly constructed in Theorem 1 when $\left\|E_{\Delta} k_{i}\right\|^{2}$ is absolutely continuous), we will have (using the Hilbert resolvent identity)

$$
\begin{aligned}
P_{\xi}\left(P_{i} f, P_{i} f\right) & =\lim _{\eta \downarrow 0} \frac{\eta}{\pi}\left((L-\xi-i \eta)^{-1} P_{i} f,(L-\xi-i \eta)^{-1} P_{i} f\right) \\
& =\frac{1}{2 \pi i} \lim _{\eta \downarrow 0} \int_{\sigma}\left\{\frac{1}{v-\xi-i \eta}-\frac{1}{v-\xi+i \eta}\right\} \mathscr{S}_{i} f(\xi) \overline{\mathscr{S}_{i} f(\xi)} d \mu_{i}(\xi),
\end{aligned}
$$

- where $\mu_{i}(\Delta)=\left\|E_{\Delta} k_{i}\right\|^{2}$. This relation implies that $P_{\xi}\left(P_{j} f, P_{j} f\right)=0$ a.a. $\xi \in \sigma(L)$, and the theorem is therefore proved since $P_{\xi}(f, f)=\sum_{i} P_{\xi}\left(P_{i} f, P_{i} f\right)$.

If we specialize to the operator $L$ we obtain, using the Cauchy residue theorem with $C$ a sufficiently large circle enclosing the spectrum of $L$

$$
\begin{aligned}
\int_{\sigma(L)} & P_{\xi}(x, y) d \xi=\frac{1}{2} \int_{C} \frac{E(l, x) \overline{E(l, y)}}{x-y} d l \\
= & \int_{a}^{b} \int_{\sigma(L)} g(v, \mu) d v\left(\frac{1}{\mu-x} \frac{1}{\mu-\bar{y}}\right) d \mu \\
= & \int_{a}^{b} k(\mu) \frac{1}{\mu-x} \frac{1}{\mu-y} d \mu=\left(\frac{1}{\mu-x}, \frac{1}{\mu-y}\right)_{L_{2}(k)} .
\end{aligned}
$$

As a final remark, we point out that the cited work of Putnam together with the Kato-Kuroda-Rosenblum perturbation theorem allows us to apply the results developed here to certain situations in which the range of the commutator $[U, V]$ is infinite dimensional. 


\section{BIBLIOGRAPHY}

1. F. Mautner, On eigenfunction expansions, Proc. Nat. Acad. Sci. U.S.A. 39 (1953), 49-53.

2. N. Dunford and J. Schwartz, Linear opsrators, Vol. 2, Interscience, New York, 1963.

3. L. Gårding, Applications of the theory of direct integrals of Hilbert spaces to some integral and differential operators, Inst. Fluid Dynamics, University of Maryland, College Park, Md., 1954.

4. W. Bade and J. Schwartz, On abstract eigenfunction expansions, Proc. Nat. Acad. Sci. U.S.A. 42 (1956), 519-525.

5. E. C. Titchmarsh, Introduction to the theory of Fourier integrals, Oxford Univ. Press, New York, 1937.

6. N. I. Achieser, Theory of approximation, Ungar, New York, 1956.

7. E. Hille, Analytic function theory, Vol. 2, Ginn, New York, 1962.

8. M. H. Stone, Linear transformations in Hilbert space, Amer. Math. Soc. Colloq. Publ. Vol. 15, Amer. Math. Soc., Providence, R. I., 1932.

9. H. Meschkowski, Hilbertsche Räume mit Kernfunction, Springer, Berlin, 1962.

10. J. von Neumann, Collected works, Vol. 3: Rings of operators, Pergamon, Oxford, 1961;

On rings of operators, reduction theory, Ann. of Math. (2) 50 (1949), 401-485.

11. J. D. Pincus, On the spectral theory of a singular integral operators, Trans. Amer. Math. Soc. 113 (1964), 101-128.

12. I. M. Gelfand and A. G. Kostjutschenko, On eigenfunction expressions associated with differential and other operators, Dokl. Akad. Nauk SSSR 103 (1955), 349-352. See also I. M. Gelfand and G. E. Silov, Verlaglgemeinerte Funktionen, Vol. 3, VEB Deutscher Verlag der Wissenschaften, Berlin, 1964; pp. 177-179.

13. Xa-Dao-Xeng (Hsia Tao-Hsing), On non-normal operators, Chinese Math. 3 (1963), 232-246.

14. C. R. Putnam, Commutators, absolutely continuous spectra, and singular integraloperators, Amer. J. Math. 96 (1964), 310-316.

15. P. Lax, Symmetrizable linear transformations, Comm. Pure Appl. Math. 7 (1954), 633-647.

16. N. I. Muskhelishvili, Singular integral equations, Noordhoff, Groningen, 1953.

17. W. Koppelman and J. D. Pincus, Spectral representations for finite Hilbert transformation, Math. Z. 71 (1959), 399-407.

18. I. P. Natanson, Theory of functions of a real variable, Ungar, New York, 1955.

19. N. Aronszajn and W. F. Donoghue, Jr., On exponential representations of analytic functions in the upper half-plane with positive imaginary part, J. Analyse Math. (1956-1957), 321-388.

BrookHAVEN NATIONAL LABORATORY, UPTON, L. I., NEW YORK 